\title{
Clinical usefulness of bedside intracranial morphological monitoring: mobile computerized tomography in the neurosurgery intensive care unit
}

\author{
Report of three cases
}

\author{
Thorsteinn Gunnarsson, M.D., M.Sc., And Jan Hillman, M.D., Ph. D. \\ Department of Neurosurgery, University Hospital, Linköping, Sweden
}

\begin{abstract}
The practice of modern neurointensive care is based on the use of multimodality monitoring to respond rapidly to physiological, biochemical, or morphological changes and avoid secondary brain injury. Until recently, one important monitoring method, computerized tomography (CT), has not been available for bedside use. The authors have over 3 years of experience with the routine use of bedside CT scanning and have developed their own method of scanning the patients in their beds. In this report, they describe three illustrative cases in which the mobile CT scanner was of great value in the management of difficult neurosurgical intensive care problems. It is concluded that the availability of bedside morphological monitoring in the neurosurgery intensive care unit is of great help in management and clinical decision making.
\end{abstract}

KEY WORDS - mobile computerized tomography - neuromonitoring • intensive care unit

Over the last two decades neurointensive care practice has developed rapidly, and the monitoring of various physiological and biochemical parameters has become routine. ${ }^{6,9,12,16}$ Until recently, one important monitoring modality, CT scanning of the head, was not available for the bedside use. ${ }^{5,11}$ We have more than 3 years experience in routine NICU bedside CT scanning, and we now perform approximately 200 bedside CT studies each year.

We have previously shown that bedside CT scanning increases patient safety and reduces the staff workload. ${ }^{11}$ In this report, we describe three cases in which the use of repeated bedside CT scanning was of great help in the management of patients with different diseases.

\section{METHODS AND SUMMARY OF CASES}

\section{Mobile CT Scanning in the NICU}

Mobile CT scanning is now a routine procedure in our NICU. The mobile CT scanner (Tomoscan M; Philips Medical Systems Findhoven, The Netherlands) has previously been described in detail as well as the scanning procedure. ${ }^{5,11}$ The videoclip shows the scanning procedure (Video Clip).

\footnotetext{
Abbreviations used in this paper: $\mathrm{CCP}=$ cerebral perfusion pressure; $\mathrm{CT}=$ computerized tomography GCS = Glasgow coma scale; $\mathrm{ICP}=$ intracranial pressure; NICU = neurosurgical intensive care unit; $\mathrm{SAH}=$ subarachnoid hemorrhage; $\mathrm{TCD}=$ transcranial Doppler.
}

\section{Click here to view Video Clip.}

Video Clip. The mobile CT scanner gantry is easy to move even in restricted areas. In the NICU, clinically unstable patients are positioned with their heads toward the center of the room; all monitoring equipment and the ventilator are mounted on the wall (at the foot end). A fiberplastic board is slid between the mattress and the bed frame and is secured with a bolt. The patient is lifted onto the headrest. To protect the staff and other patients in the NICU from scattered radiation, mobile lead shields are used. The radiology assistants have the responsibility of checking the final position and performing the scanning. The images can be directly compared with previous studies from the hospital's digital x-ray film archive on double computer screens in the NICU.

\section{Case 1: A Child With Isolated Head Injury}

This 9-year-old boy, a front seat passenger wearing a seatbelt, suffered trauma to his head in the right temporal area during a motor vehicle accident. At the scene of the accident he was judged to have a GCS score of 4 to 5 . At the scene of the accident he was intubated and a CT scan, obtained at the referring hospital, revealed a depressed skull fracture in the right temporal bone, one significant temporal contusion, and multiple small contusions in both the hemispheres.

On admission to our department, the patient had symmetric reactive pupils and was taken to the operating room immediately for elevation of the depressed skull fracture, removal of the temporal lobe contusion, cranioplasty, and insertion of an intraventricular drain for the measurement of ICP. Postoperatively, the patient's ICP was 3 to $4 \mathrm{~mm}$ 

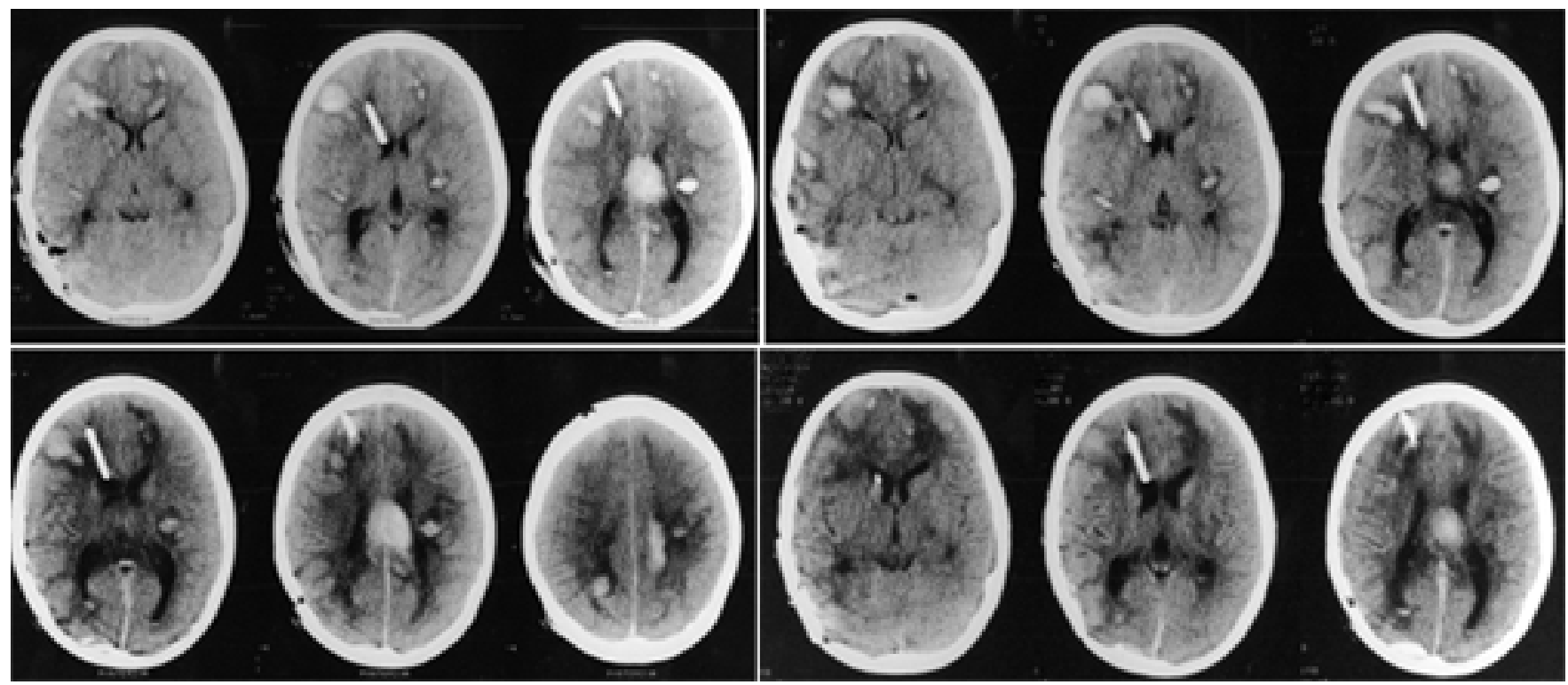

Fig. 1. Case 1. Bedside CT studies. Despite very low CPP values and coagulation problems, no general infarctions or further bleedings occurred, as demonstrated by postoperative bedside CT scans on Day 1 (upper left), Day 6 (upper right), Day 10 (lower left), and Day 15 (lower right).

$\mathrm{Hg}$ and within hours it increased to $50 \mathrm{~mm} \mathrm{Hg}$. A postoperative mobile CT scan demonstrated the development of multiple contusions cortically and centrally located, and a barbiturate coma was induced (Fig. 1 upper left). During the postoperative course, the patient was clinically unstable; his ICP was high (rising over $50 \mathrm{~mm} \mathrm{Hg}$ ) and there were long periods in which his CPP was approximately 40 $\mathrm{mm} \mathrm{Hg}$.

The postoperative course was complicated both by renal failure, which was treated with peritoneal dialysis, and by coagulopathy resulting from the formation of antibodies to coagulation factors. We also suspected meningitis, although cultures were negative for this. During this period, the patient had 11 drung infusion pumps, reflecting his clinical instability, and an intraventricular catheter, a peritoneal dialysis catheter, jugular bulb catheter, central venous catheter, urinary catheter, and a gastric tube were placed. The patient was discharged to the referring hospital ICU on Day 19 and later underwent placement of a ventriculoperitoneal shunt after the development of hydrocephalus.

During the stay in the NICU, the patient underwent a total of 12 CT scanning procedures of the head. Despite the low CPP and other complications, the repeated scans demonstrated no development of infarction, hydrocephalus, or additional contusions and, as expected, reduction of the brain edema (Fig. 1).

\section{Case 2: A Multitrauma Patient}

This 21-year-old woman was referred after being involved in a severe traffic accident. On the scene of the accident, the patient was breathing spontaneously with decerebrating movements in all four limbs (GCS score of 4) and small-sized pupils.

A CT scan obtained at the referring hospital revealed thin, left-sided extracerebral bleeding with compression of the ventricle system and a midline shift of $0.5 \mathrm{~cm}$ with no contusions or skull fractures. Computerized tomography and radiography of the spine demonstrated a fracture through the base of the dens with a 5-mm dislocation of the lower fragment (Fig. 2). There was also a fracture without dislocation on the right lateral mass of the C-1. Fractures in the ventral part of the T-5 vertebral body and in L-2, without compression of the spinal canal, were also seen. Additionally, the patient had a stable fracture in the os ileum and a two fractures in the left arm.
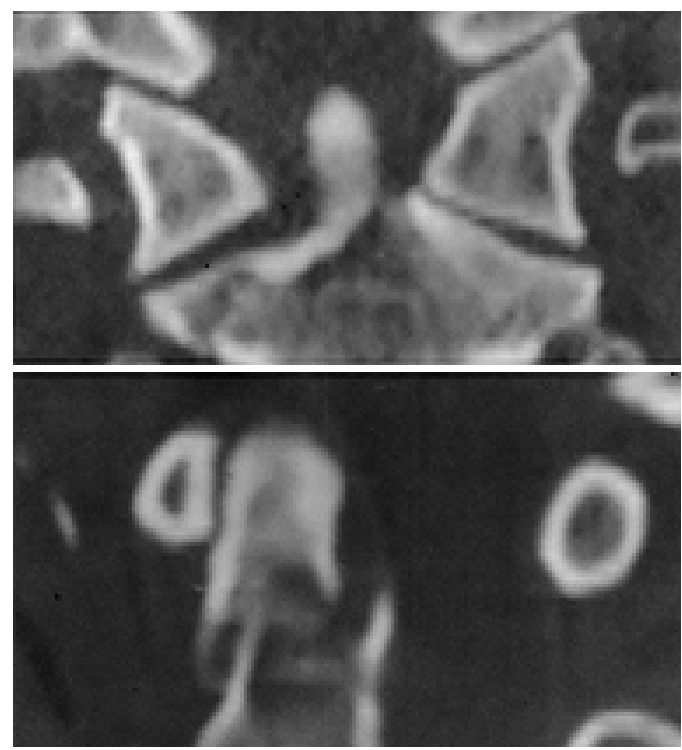

Fig. 2. Case 2. Reconstrucion of CT scans demonstrating the fracture of the dens with the fracture line through the base of the dens (upper) and dislocation of the lower fragment into the spinal canal (lower). 

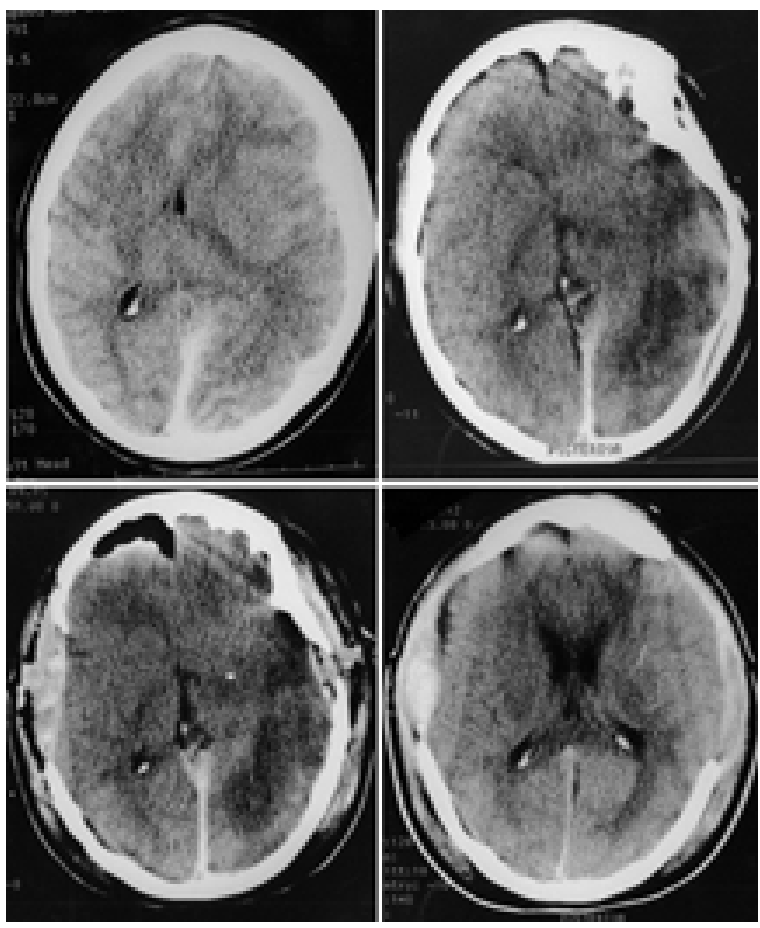

Fig. 3. Case 2. Upper Left: The first CT scan obtained after admission to our hospital demonstrating a traumatic $\mathrm{SAH}$ and brain swelling with midline shift. Upper Right: Bedside CT scan obtained on Day 3 after the insertion of an intraparencymal ICPmeasuring transducer and bilateral craniectomies. Lower Left: Bedside CT scan obtained on Day 6. Lower Right: Bedside CT scan obtained on Day 13 revealing reduction in the brain swelling and reduction of the edema in the occipitotemporal area.

After admission to our department, a new CT scan revealed a progression of the brain edema, a suspected contusion, and a traumatic SAH on the left side (Fig. 3 upper left). It also demonstrated pneumothorax and right-sided lung contusions. The patient was taken to the operating room where a chest drain and an intraparencymal ICP measuring transducer were inserted. Barbiturate coma was induced, and methylprednisolone was given because of suspected spinal cord injury. ${ }^{3}$ During the following day, the ICP remained stable $(11-13 \mathrm{~mm} \mathrm{Hg})$ but thereafter increased rapidly to 30 to $35 \mathrm{~mm} \mathrm{Hg}$ despite aggressive treatment. Repeated CT scans of the head revealed areas of suspected infarction/edema in the occipitoparietal area and midline shift but no bleeding or hydrocephalus. At that time, the coagulation parameters were highly abnormal, and this persisted for more than 2 weeks. On the 4th day of increasing ICP, bilateral craniectomies and duraplasty were performed in effort to control the ICP. For the next 5 days, the CPP was only 40 to $50 \mathrm{~mm} \mathrm{Hg}$ with prolonged periods approaching $30 \mathrm{~mm} \mathrm{Hg}$ despite massive inotropic and volume therapy. The patient was septic. Two weeks after admission and the time at which this paper was submitted, the patient was in our NICU and was gradually stabilizing. During the most intensive period, the patient had nine drug infusion pumps, as well as placement of pulmonary artery catheter, central venous catheter, an intraparencymal ICP transducer, trache- ostomy, urinary catheter, and a gastric tube. Fourteen consecutive CT scans have demonstrated general brain swelling with infarction/edema in the left occipitotemporal region, but no signs of general ischemia and infarction, and a good demarcation between gray and white matter remains present throughout the brain. During the last few days, reduction in the brain edema has been observed (Fig. 3).

\section{Case 3: A Woman With SAH}

This 65-year-old woman was referred after suffering an SAH. She was initially drowsy and disoriented, and CT scanning revealed SAH with blood in the Sylvian fissure as well as hydrocephalus. She rapidly deteriorated, was intubated, and transferred to this department. After arrival, an intraventricular drain was inserted and cerebral angiography revealed an anterior communicating artery aneurysm that was clipped. The following day, the patient was still treated in a respirator; her GCS score was 8. The clinical course was very unstable with cardiovascular instability, pneumonia, and later suspected meningitis. During this period, on Day 11, TCD ultrasonography of the right middle cerebral artery detected a rise in blood flow from 70 to $80 \mathrm{~cm} / \mathrm{second}$ to $130 \mathrm{~cm} / \mathrm{second}$ and her neurological condition deteriorated (GCS score of 7). Despite many attempts, a TCD signal could never be detected from the left middle cerebral artery. The patient was treated with nimodipine, and hypertensive, hypervolemic, hemodilutional therapy. ${ }^{16}$ The high blood flow velocity gradually decreased. Despite the patient's poor neurological condition, partly due to the initial SAH and partly due to possible vasospasm, no signs of infarction were seen on repeated CT scans. The patient was discharged on Day 19 to the ICU of the referring hospital for further treatment. She later received a shunt for communicating hydrocephalus (Fig. 4) and made a good recovery.

\section{DISCUSSION}

Reported clinical and laboratory data have demonstrated the vulnerability of the injured brain during perturbations in physiological parameters. ${ }^{1,7-9,15,17}$ Neurointensive therapy should be practiced on a minute-to-minute basis, and the patient should be manipulated as little as possible to avoid exacerbating clinical instability. Neurointensive care has been shown to improve outcome. ${ }^{21}$ Today, multimodality neuromonitoring is mandatory in the NICU, including physiological and biochemical parameters as well as repeated evaluation of intracranial morphological changes. ${ }^{12,16,18}$

Computerized tomography scanning of the head is the standard method for such evaluation, and because of the dynamic processes involved in traumatic neurological injury, the use of serial CT investigation has been recommended in the literature. ${ }^{18}$ In most hospitals, however, the CT scanner is situated in the radiology department, and the patient has to be transferred from the NICU. Our previous study and data reported in similar studies confirm the dangers of intrahospital transportation., ${ }^{2,4,10,13,14,19,20} \mathrm{We}$ found that transportation of medium- and high-risk patients was associated with medical or technical complications in 20 to $25 \%$ of the cases. Moving unstable patients 


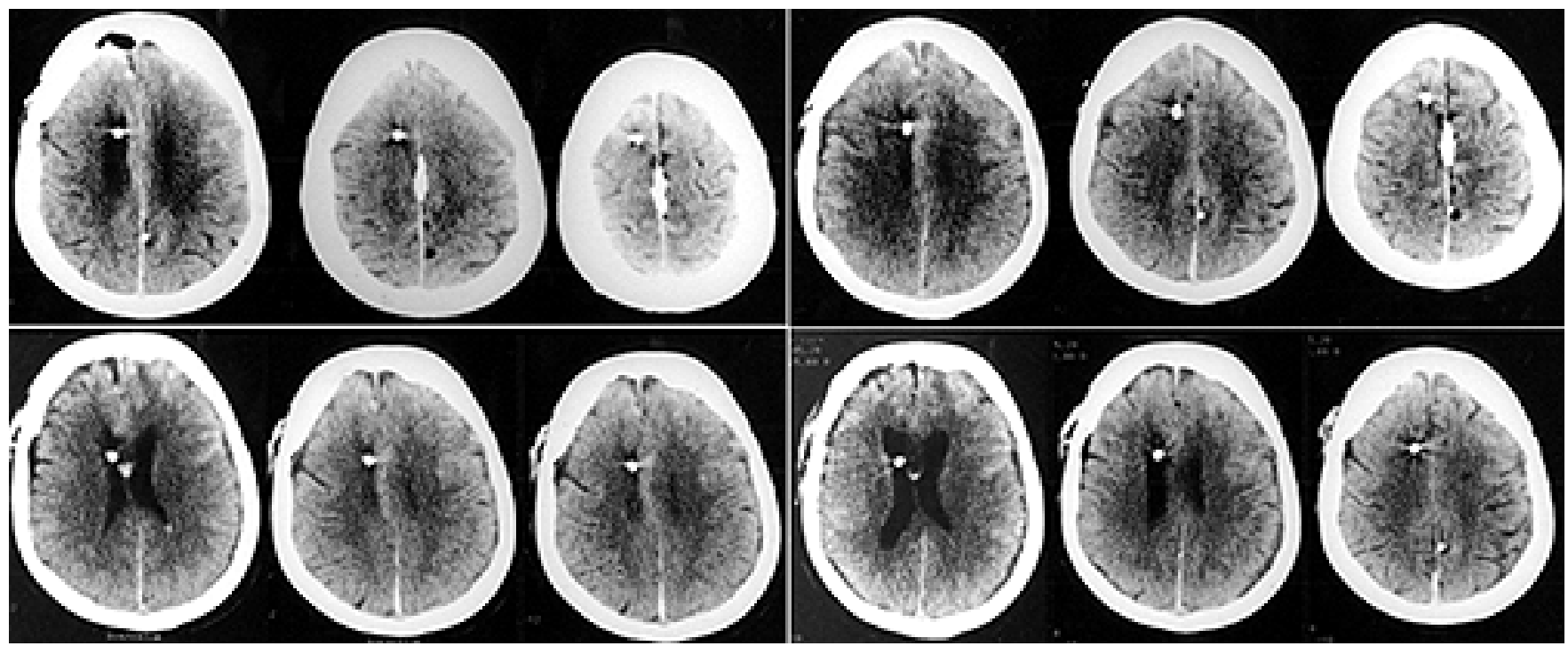

Fig. 4. Case 3. Bedside postoperative CT scans obtained on Day 1 (upper left), Day 3 (upper right), Day 6 (lower left), and Day 11 (lower right). Despite poor neurological status and high TCD values, CT scanning demonstrated no signs of infarction.

from the controlled environment of the NICU for CT scanning seems almost unacceptable in this perspective, although this is performed on daily basis in most neurosurgical departments.

As a consequence, the inherent risks of transportation for CT scanning of unstable patients may often cause few or fewer CT studies to be performed during the clinical course of individual patients, and important information may be lost. Repeated morphological evaluation not only serves to reveal surgically accessible lesions but also helps in the interpretation of ongoing disturbances in physiological and biochemical parameters, thus supporting the clinical decision-making process.

The introduction of the mobile CT scanner is an integral part of an effort in our center to develop the concept of bedside patient testing, including all levels of multimodality monitoring. ${ }^{12}$

The three patients described in this report present everyday cases for the practicing neurosurgeon and have been chosen to illustrate some of the benefits of having the mobile CT scanner.

The patient in Case 1 had an unstable clinical course; his ICP was high and CPP was low (approximately $40 \mathrm{~mm}$ $\mathrm{Hg}$ ) for prolonged periods of time. Because of the 11 pump infusions of drugs and the placement of an endotracheal tube and numerous catheters, the risk of a physiological deterioration or a technical mishap during the transportation was high. ${ }^{2,4,9,11,13,19,20}$ In the case of complicating events that develop outside the NICU, all therapeutic interventions are more difficult to accomplish. In our case the occurrence of coagulopathy and numerous contusions makes frequent updating of the morphological information of major importance. The absence of both infarction and expansion of the contusions were key observations in guiding treatment.

Similar problems were faced in Case 2; the patient was physiologically unstable and she developed coagulopathy. This case also illustrates that the frequent acquisition of morphogical information is of major importance in determining the brain's tolerance to low CCP values. On the basis of the physiological data alone, and in light of all complicating factors, one may have considered withdrawing treatment. With access to low-risk repeated CT scanning, the absence of widespread infarction and the reduction of brain edema, despite the high ICP and the low CPP values, were crucial determinants in our decision to maintain therapy. The spinal fractures in Case 2, which had not yet been treated because of the patients' clinical condition, further underscore the importance of manipulating the patient as little as possible.

As in Case 3, the diagnosis of brain ischemia/infarction from vasospasm is crucial in poor-grade patients with SAH. Clinical signs and TCD ultrasonography demonstrated delayed ischemic complications. This patient was also vulnerable to complication during transportation. Repeated mobile CT scans revealed no signs of infarction, again increasing our confidence that the hypertensive, hypervolemic, hemodilutional therapy was effective.

The patients in Cases 2 and 3 suffered ongoing infections, and transportation from the closed NICU environment, had it been done, might have led to spread of bacteria, which is of particular concern for patients infected by such entities as methicillin-resistant staphylococci and tuberculosis. Because of its greater user friendliness, mobile CT scanning can also easily be performed in small isolation rooms, thus contributing one additional advantage to this system.

This technique of morphological monitoring has the potential of being transformed into instruments for physiological monitoring through the xenon CT blood flow technology. This possibility would seem to be of great value in the mobile CT scanner setting.

\section{CONCLUSIONS}

We have shown the value of obtaining frequent, repeat- 
ed, low-risk CT scans not only in detecting surgical lesions but also the ongoing physiological and biochemical changes. Assessment of these parameters leads to an increased level of confidence in the management of patients with intracranial disease. The results from this report will be studied further in a prospective manner in order to analyze the impact of mobile CT availability on the clinical decision-making process in individual patients.

\section{Acknowledgments}

The authors thank Dr. Håkan Lindstam for help with the selection of figures, as well as the nusing staff in the NICU for active participation in the preparation of this manuscript.

\section{References}

1. Andersen BJ, Unterberg AW, Clarke GD, et al: Effect of posttraumatic hypoventilation on cerebral energy metabolism. J Neurosurg 68:601-607, 1988

2. Andrews PJD, Piper IR, Dearden NM, et al: Secondary insults during intrahospital transport of head-injured patients. Lancet 335:327-330, 1990

3. Bracken MB, Shepard MJ, Holford TR, et al: Administration of methylprednisolone for 24 or 48 hours or tirilazad mesylate for 48 hours in the treatment of acute spinal cord injury. Results of the Third National Acute Spinal Cord Injury Randomized Controlled Trial. National Acute Spinal Cord Injury Study. JAMA 277:1597-1604, 1997

4. Braman SS, Dunn SM, Amico CA, et al: Complications of intrahospital transport in critically ill patients. Ann Intern Med 107:469-473, 1987

5. Butler WE, Piaggio CM, Constantinou C, et al: A mobile computed tomographic scanner with intraoperative and intensive care unit applications. Neurosurgery 42:1304-1311, 1998

6. Chan KH, Dearden NM, Miller JD, et al: Multimodality monitoring as a guide to treatment of intracranial hypertension after severe brain injury. Neurosurgery 32:547-553, 1993

7. Cortbus F, Jones PA, Miller JD, et al: Cause, distribution and significance of episodes of reduced cerebral perfusion pressure following head injury. Acta Neurochir 130:117-124, 1994

8. DeWitt DS, Jenkins LW, Prough DS: Enhanced vulnerability to secondary ischemic insults after experimental traumatic brain injury. New Horiz 3:376-383, 1995

9. Enblad P, Persson L: Impact on clinical outcome of secondary brain insults during the neurointensive care of patients with subarachnoid haemorrhage: a pilot study. J Neurol Neurosurg Psychiatry 62:512-516, 1997
10. Gervais HW, Eberle B, Konietzke D, et al: Comparison of blood gases of ventilated patients during transport. Crit Care Med 15:761-763, 1987

11. Gunnarsson T, Theodorsson A, Karlsson P, et al: Mobile computerized tomography scanning in the neurosurgery intensive care unit: increase in patient safety and reduction of staff workload. J Neurosurg 93:432-436, 2000

12. Hillman J: Biochemical monitoring in neuro-intensive caretoday and tomorrow. A review, in D'Orazio P, Fogh-Andersen N, Larsson L (eds): The Confluence of Critical Care Analysis and Near Patient Testing. Proceedings of the 17th International Symposium. Madison, WI: Omnipress, 1998, pp $132-138$

13. Indeck M, Peterson S, Smith J, et al: Risk, cost, and benefit of transporting ICU patients for special studies. J Trauma 28: 1020-1025, 1988

14. Kollef MH, Von Harz B, Prentie D, et al: Patient transport from intensive care increases the risk of developing ventilator-associated pneumonia. Chest 112:765-773, 1997

15. Marmarou A, Anderson RL, Ward JD, et al: Impact of ICP instability and hypotension on outcome in patients with severe head trauma. J Neurosurg 75:S59-S66, 1991

16. Martin N, Khanna R, Rodts G: The intensive care management of patients with subarachnoid hemorrhage, in Andrews BT (ed): Neurosurgical Intensive Care. New York: McGrawHill, 1993, pp 291-310

17. Piek J, Chesnut RM, Marshall LF, et al: Extracranial complication of severe head injury. J Neurosurg 77:901-907, 1992

18. Servadei F, Nasi MT, Giuliani G, et al: CT prognostic factors in acute subdural haematomas: the value of the 'worst' CT scan. Br J Neurosurg 14:110-116, 2000

19. Smith I, Fleming S, Gernaianu A: Mishaps during transport from the intensive care unit. Crit Care Med 18:278-281, 1990

20. Waddel G: Movement of critically ill patients within hospital. Br Med J 2:417-419, 1975

21. Warme PE, Bergstrom R, Persson L: Neurosurgical intensive care improves outcome after severe head injury. Acta Neurochir 110:57-64, 1991

Manuscript received September 18, 2000.

Accepted in final form October 10, 2000.

The authors have not received any financial compensation for this work and have no financial interest in the device described in this paper.

Address reprint requests to: Thorsteinn Gunnarsson, M.D. M.Sc., Department of Neurosurgery, University Hospital, 58185 Linköping, Sweden. email: thorsteinn.gunnarsson@lio.se. 